\title{
An Experimental Procedure to Obtain the Equilibrium Contact Angle from the Wilhelmy Method
}

\author{
C. Della Volpe', D. Maniglio', S. Siboni' and M. Morra² \\ 1 University of Trento, Department of Materials Engineering, Via Mesiano 77, Trento, I - Italy \\ 2 NobilBio, Villa Franca d'Asti (AT), Str. S. Rocca 32, 1 - Italy \\ e-mail: devol@devolmac.inz.unitn.it
}

\begin{abstract}
Résumé - Une méthode expérimentale pour mesurer l'angle de contact d'équilibre avec la technique de Wilhelmy — La théorie des angles de contact a été développée en utilisant le concept d'angle de Young, une relation d'équilibre établie pour des surfaces complètement lisses et homogènes ; sur surfaces réelles, on considère habituellement que l'on peut obtenir des états «métastables » d'équilibre dans lesquels la forme du ménisque le long de la ligne à trois phases n'est pas complètement équivalente à celle du ménisque d'équilibre.

Dans cet article, nous présentons une nouvelle méthode d'obtention de l'angle de contact d'équilibre par une simple modification de la microbalance de Wilhelmy. L'apport d'énergie acoustique au liquide à une fréquence capable de provoquer la formation résonante d'ondes capillaires, suivi par la réduction de l'amplitude de la vibration, permet au ménisque de bouger et de passer de sa forme métastable, d'avancée ou de recul, à celle d'équilibre stable.

Ce résultat paraît être essentiellement indépendant des conditions initiales ; il a été possible de confirmer la théorie selon laquelle les barrières d'énergie entre états métastables loin de l'équilibre sont plus faibles que celles à l'équilibre.

L'équation qui met en relation le cosinus de l'angle de contact d'équilibre et la moyenne arithmétique des cosinus des angles d'avancée et de recul a été confirmée pour des surfaces homogènes, mais elle a été réfutée dans le cas de surfaces macroscopiquement hétérogènes.

Cette méthode paraît par ailleurs prometteuse par sa simplicité et son faible coût.

Mots-clés : angle de contact d'avancée, angle de contact de recul, hystérésis, angle de contact d'équilibre, vibrations, ondes capillaires.

\footnotetext{
Abstract - An Experimental Procedure to Obtain the Equilibrium Contact Angle from the Wilhelmy Method - The theory of contact angles has been developed using the concept of the Young contact angle, an equilibrium quantity related to perfectly smooth and homogeneous surfaces; on real surfaces it is commonly accepted that one can obtain "metastable" equilibrium states, in which the shape of the meniscus along the triple line is not fully equivalent to the "equilibrium" meniscus.

In the present paper, a new method is presented to obtain an equilibrium contact angle with a simple modification of the Wilhelmy microbalance. The supply of acoustic energy to the liquid at a frequency which induces the resonant formation of capillary waves, followed by the reduction of the amplitude of the vibration, allows the meniscus to move from the metastable advancing or receding shape to the stable equilibrium one.
} 
This result appears to be significantly independent of the initial conditions; it has been possible to confirm the theoretical provision that the energy barriers between metastable states far from equilibrium are lower than the barriers at equilibrium.

The equation which relates the equilibrium cosine of the contact angle and the mean of advancing and receding cosines has been confirmed on homogeneous surfaces, but has been disproved on macroscopically heterogeneous surfaces.

The method appears promising also for its simplicity and low cost.

Keywords: advancing contact angle, receding contact angle, hysteresis, equilibrium contact angle, vibrations, capillary waves.

\section{INTRODUCTION}

The contact angle can be theoretically defined by the famous Young equation, written in 1805 [1]:

$$
\gamma_{s v}=\gamma_{l v} \cos \theta+\gamma_{l s}
$$

As a ratio of functions of state, it is indeed a function of state. The surfaces considered in the previous equation are perfectly smooth and homogeneous and the state of the system is an equilibrium state. In a real experimental situation, it is practically impossible to have perfectly smooth and homogeneous surfaces or to be in a true equilibrium state; as a consequence, Young angle is an ideal quantity. Indeed, in experimental situations the meniscus profile is not fully equivalent to the "equilibrium" meniscus.

A common interpretation of this phenomenon has been developed by Johnson and Dettre [2] and it is expressed by the qualitative graph of Figure 1. A real interfacial system is for many reasons [2-5] not in its lowest energy state, corresponding to the lowest minimum of the shown curve; its real state will correspond to a local minimum on a side of the curve, i.e. a metastable equilibrium state.

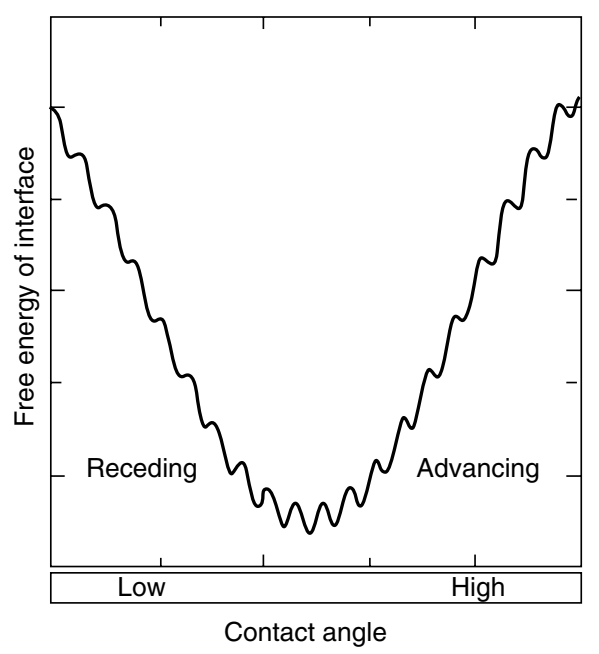

Figure 1

The surface free energy of the triphase system $v s$. the experimental contact angle; the shape of the local minima is important in its effect on the real "mobility" of the meniscus.
The measured contact angle is higher or lower than the equilibrium contact angle and is commonly called advancing or receding, depending on the zone of the shown curve in which the system really rests and which depends on the moving direction of the obtained meniscus at its formation; the difference between the extreme values is called hysteresis.

This fact can simply explain the wide differences present in literature among the results obtained by different researchers even on the same surface [6]. Given that it is impossible to develop a meniscus at "zero" speed, many researchers allow the meniscus to "relax" simply stopping every movement and waiting a certain time. But it has never been proven that the meniscus so obtained was not entrapped in a local free energy minimum.

Many theoretical works have been performed to explain and to analyze the situation [7-18], exploring the presence of different causes of hysteresis.

On the contrary, a very few cases of experimental trials dealing with the subject of equilibrium state in contact angle measurements are present in our knowledge in literature [1924 ], while many other papers deal with the argument of experimental hysteresis [25-28].

In the oldest papers, the researchers have simply applied what Fowkes and Harkins called the slight shaking technique [19-21] to faster obtain the equilibrium meniscus shape. In very few recent papers, direct vibrations obtained by mechanical or acoustical methods have been applied to the solid surfaces [22-24], studying the results along with the variation of the defects present on the surface or with the characteristics of single "spike-like" movements.

In these cases, a general relation of the form:

$$
\cos \theta_{\text {equ }}=0.5 \cos \theta_{\text {adv }}+0.5 \cos \theta_{\text {rec }}
$$

has been obtained, in which the indices indicate the equilibrium state, the advancing and receding angles, respectively.

Equation (2) is referred to the advancing and to the receding contact angles; on the contrary, the most famous equations obtained by Wenzel [29] and Cassie and Baxter [7, 8] refer to the "equilibrium" values and can generally be expressed by:

$$
\cos \theta_{\text {mean }}=\sum_{i} r_{i} f_{i} \cos \theta_{\text {equ }, i}
$$


where the index "mean" corresponds to the equilibrium value on rough or heterogeneous surfaces and the $i$ to the different surface-patches present; $r$ and $f$ are coefficients expressing the role of roughness and heterogeneity, respectively. In Equation (2), we have a linear combination of contact angle cosines as in Equation (3), but the considered quantities are not equilibrium ones.

Even Johnson and Dettre [2, 10, 25] used to compare the advancing and receding contact angles with the result of Cassie and Baxter [7, 8] calculations. In doing so, it is clear that the two equations appear conflicting because Equation (2) would impose a fixed ratio $(=1)$ between the different components of the surface. A partial justification is that Johnson and Dettre [2, 10, 25] considered the advancing and receding values as mainly representative of the two different portions of the analyzed surface (low surface energy and high surface energy portions, respectively).

However, they have proposed [28] later a different equation which makes clearer the relation among all these quantities: this equation expresses the relative surface coverage index, $I$, as:

where:

$$
I=100 \frac{\langle\cos \theta\rangle-\cos \theta_{\min }}{\left\langle\cos \theta^{0}\right\rangle-\cos \theta_{\min }}
$$

$$
\begin{aligned}
\langle\cos \theta> & =\left(0.5 \cos \theta_{\mathrm{adv}}+0.5 \cos \theta_{\mathrm{rec}}\right) \\
\left\langle\cos \theta^{0}>\right. & =\left(0.5 \cos \theta_{\mathrm{adv}}^{0}+0.5 \cos \theta_{\mathrm{rec}}^{0}\right)
\end{aligned}
$$

The indices "adv" and "rec" correspond to the advancing and receding angles, the index "min" and exponent " 0 " denote the values of the two kinds of surfaces, constituting the total patchwork. So, Johnson and Dettre [28] have in fact considered the validity of both equations, contemporarily, but without any justification of Equation (2).

The comparison of the theoretical results with the experimental ones for Equation (2) has been made, de facto, using only the data by Andrieu et al. [23]; this paper appears, in our knowledge, as the unique wide set of experimental data obtained by "vibrational" methods. Those data have been obtained by a modified sessile drop method on heterogeneous surfaces made of polyethylene terephtalate (PET) added with macroscopic drops of more hydrophobic materials and by few other polymers (Fig. 4 in [23]).

One can easily note that the straight line $y=x$, i.e. the left vs. right portion of Equation (2), does not fit well the data and the calculated $\chi^{2}$ appears quite high. Particularly, the data obtained by the methylene iodide liquid on the same kind of surfaces as water are completely out of the chosen fitting. The best-fit curve can be easily calculated and it corresponds to something as:

$$
<\cos \theta>=\left(0.3 \cos \theta_{\mathrm{adv}}+0.7 \cos \theta_{\mathrm{rec}}\right)
$$

which is different from Equation (2). The use of the same samples for the two liquids can however help to exclude a significant role of the sample roughness.
In the present paper, we show the results obtained positioning a loudspeaker under the container of the liquid in a Wilhelmy microbalance and producing an acoustic frequency corresponding to the resonant formation of capillary waves at the liquid surface. Then, slowly reducing to zero the amplitude of vibration, we have put the system into its lowest energy minimum. We have found that the same absolute minimum can be attained from advancing and receding menisci, independently of the initial conditions of formation. This minimum is frequently, but not always, in agreement with the previous Equation (2); an interpretation of this disagreement is furnished.

Some samples of common materials have been studied varying the speed and the percentage of heterogeneity. We have also recalculated the solid surface free energy of polypropylene (PP) from some of the obtained "equilibrium" data. The method appears as a promising one to study the energetics of solid surfaces; in fact, in our opinion, the numeric value of the contact angle obtained at equilibrium is the natural candidate for the evaluation of the surface free energy of a material.

\section{MATERIALS AND METHODS}

The experiments were performed using a Cahn microbalance DCA 322 which is able to collect data at a speed of $1 \mathrm{~Hz}$. (It has to be noted that the low speed in collecting data can work as a kind of lowband filter with respect to the effective force values, due to the higher frequency of the used acoustic vibration.) To the balance it was added a vibration apparatus composed by a loudspeaker (diameter $6 \mathrm{~cm}$, nominal impedance $8 \Omega, 0.250 \mathrm{~W}$ ) driven by a function generator (model MK 1050 by Mitek). Upon the vibrating diaphragm, it was placed a plastic ring to hook a $4 \mathrm{~cm}$ Teflon beaker filled with the measure liquid. In this way, the vibrating parts were made close together to avoid unwanted or accidental movements of the becker. All the DCA runs were performed at room temperature $\left(22 \pm 2^{\circ} \mathrm{C}\right)$.

As a measure liquid, ultrapure water $\left(18.5 \mathrm{M} \Omega \mathrm{cm}^{-1}\right.$ produced by a Millipore Milli-Q device) was employed, using only fresh liquid for each experiment. The methylene iodide, $99 \%+$ was by Acros Organics (Milan, Italy). PP was in rectangular plates $(1 \times 1.5 \mathrm{~cm}$ plates, Goodfellow $)$ with a roughness evaluated by atomic force microscopy $\left(R_{a}=48 \mathrm{~nm}\right)$.

Glass microscopy slides, $24 \times 30 \mathrm{~mm}$ (by Prestige), were radio frequency $(\mathrm{RF})$ plasma treated in oxygen $\left(5^{\prime}\right.$ at $50 \mathrm{~W}$ and $20 \mathrm{cc}$ (Standard Temperature and Pressure, STP)/min flow rate, to clean it) and then submitted to different modifying treatments. Some glass samples, previously cleaned in RF-oxygen plasma, were then sprayed by a common commercial acrylic white paint for car in a different percentage.

Other plasma-treated samples were obtained by deposition from tetramethyldisiloxane (TMDSO) or propylene plasma, 
followed, except for Sample 0, by hydrophilization through air plasma treatment. Propylene and TMDSO, $>98 \%$ pure, were purchased from Aldrich. Plasma deposition and plasma treatment were performed in a parallel plate stainless steel reactor, coupled with a $13.56 \mathrm{MHz}$ RF generator. TMDSO or propylene were introduced into a glass vessel connected to the reactor with a glass valve and a glass-metal joint. The temperature of the monomer in the vessel was maintained at $25^{\circ} \mathrm{C}$ throughout the cycle by a water bath. The flow rate, calculated by the initial increase in the system pressure with time after cutting off the vacuum pump, was about $100 \mathrm{cc}$ (STP)/min. Sample 0, hydrophobic, was obtained using a deposition time of $2 \mathrm{~min}$ and a power discharge of $50 \mathrm{~W}$. Samples 1, 2 and 3 were obtained using the same deposition conditions followed by air plasma treatment for 5, 10 and 15 s respectively. Air plasma treatment was performed with a $50 \mathrm{~W}$ discharge power and $20 \mathrm{cc}$ (STP)/min flow rate.

The basic operations of the vibration method are described in the following, while some other details will be presented in Section 2.

During the advancing and receding phases, the vertical movement of the liquid container was stopped and a vibration cycle started. This cycle was composed of four temporal sequences with a time length of 30" each: a first step of quietness (self-relaxing step) during which the meniscus self-relaxed; a second step (high-vibration step) during which the Teflon beaker was subjected to a highintensity vibration by applying to the speaker a $3.6 \mathrm{~V}$ amplitude, $54 \mathrm{~Hz}$ frequency square wave signal. During the third step (freezing step), the amplitude of the square wave was progressively reduced to zero. The last step was a period of quietness during which the system eventually further relaxed (self-relaxing step). After the procedure completion, the vertical movement of the liquid container was started again. By repeating the sequence described above, we would collect, from the usual Wilhelmy graph, a set of equilibrium force points possibly aligned on a straight line, and then calculate a corresponding equilibrium angle.

The frequency of $54 \mathrm{~Hz}$ was chosen to give the liquid shape a high-intensity, slowly rotating and geometrically regular wave interference figure so to make the three-phase line move along the whole sample surface perimeter. It appeared to be the most efficient working frequency. To compare the relative effects of self-relaxing and vibrationinduced relaxing (Section 2), a self-relaxing step of $10 \mathrm{~min}$ was compared with the high-vibration step, followed by a new self-relaxing step of $10 \mathrm{~min}$.

\section{RESULTS AND DISCUSSION}

A typical result is shown in Figure 2 with the common representation force $v s$. immersion. In this particular case, we have realized at each immersion level $(4,8$ and $11 \mathrm{~mm})$ a "local" advancing and receding cycle, respectively indicated by the A, B, C and A', B', C' symbols. However, this procedure is not strictly necessary and the analysis can be realized along he common run.

The same measurement is shown in Figure 3 with the representation force $v s$. time. The sample was a PP sheet in water at room temperature.

In the force $v s$. immersion representation, all the vibration process is "invisible" and it is contained in the vertical line. As one can see, the exit from this step occurs at a certain force level which is different from the maximum (or the minimum in the case of the receding phase) value. This level

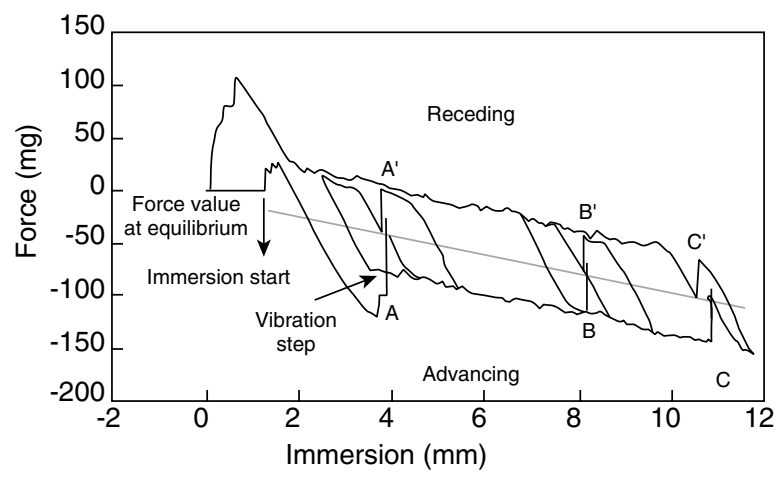

Figure 2

A typical experimental result: in this particular case, we have realized at each immersion level $(4,8$ and $11 \mathrm{~mm})$ a "local" advancing and receding cycle, respectively indicated by the A, B, C and A', B', C' symbols. However, this procedure is not strictly necessary and the receding analysis can be realized along the common run. The sample is PP in water. The line extrapolated from equilibrium points allows to calculate the contact angle from the equation $F_{\text {equ }}=\rho \gamma \cos \theta_{\text {equ }}$.

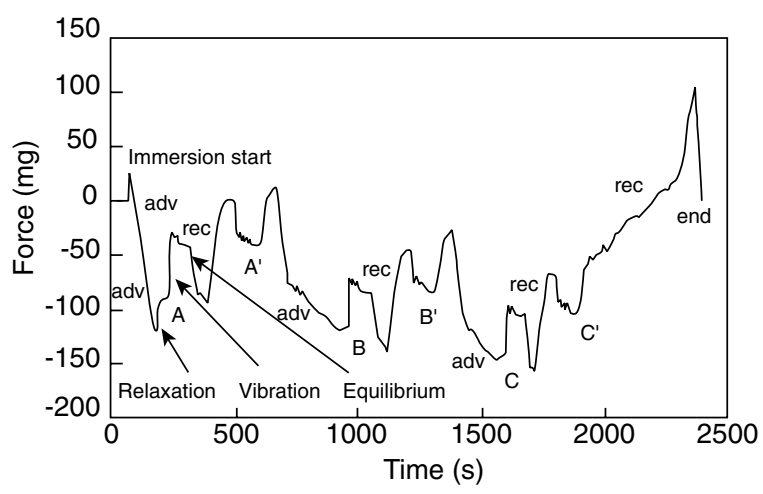

Figure 3

The same run as in Figure 2 in force vs. time representation. Note the correspondence of the different steps of the measure. 
is the actual level of the "equilibrium" meniscus and the corresponding point can be used for the extrapolation fitting of the "equilibrium" force.

In contrast, in the force $v s$. time representation, all the steps of the measurement are clearly shown. One can see the spontaneous relaxation, which slightly increases the force in the case of advancing and decreases it in the case of receding. This event occurs at each speed and indicates that the starting state is not stable. The idea that waiting for few minutes or even hours can produce the "equilibrium" meniscus via this spontaneous relaxation is probably wrong in absence of significant environmental vibrations. The system maintains memory of its past and it can remain eventually blocked in one of the many metastable states in which the environmental vibrations can push it.

The effect of the forced vibration is very strong and fast. Immediately the system starts to oscillate around the real minimum of the meniscus free energy; slowly reducing the amplitude of the given acoustic energy causes the system finally to stop in a state near the lowest minimum or exactly in it. The extrema obtained during the oscillations are not significant. In the force $v s$. immersion representation, they appear as well-defined points, but this is actually not true. On the contrary, in the force vs. time representation, it clearly appears that the extrema of the force values are simply instantaneous states of the system while the obtained final point is really stable, independent of the initial state of the meniscus, of its speed, of its advancing or receding condition, etc. In the force $v s$. immersion representation, the final states rest on the same straight line, which corresponds to a particular contact angle, and we consider it to be, possibly, the equilibrium contact angle. The six points determined in this way can be connected by a straight line parallel to those obtained by the advancing and receding processes and an "equilibrium" value of the contact angle can be calculated extrapolating at zero immersion the Wilhelmy equation, $F=\rho \gamma \cos \theta$. As a common consideration, the low number of "equilibrium" points is a limit for the precision of the measurement.

It is worth of note that in our idea the slow reduction of energy amplitude is very important to attain the equilibrium state; this aspect is particularly analyzed in the mathematical appendix.

A comparison of the effect obtained by the spontaneous relaxing of the metastable state through the environmental or thermal vibrations and that obtained by the forced vibration method is shown in Figure 4. It appears clear that the forced relaxing is exceedingly able to induce the effective relaxation of the meniscus and that the final state does not depend on the initial one, being an equilibrium state: i.e. the two final points are on the same straight line, parallel to the main advancing and receding processes (the two curved portions near the initial phase of the immersion are due to geometrical asperities of the cut sample). Moreover, the spontaneous relaxation was obtained in a much longer time than in the vibrational experiments; this is clearly shown in Figure 5 (force $v s$. time representation).

The acoustic energy supply to the interface system gives different effects whether it is in a metastable state or in a stable state. In two different experiments, we have supplied energy, increasing the amplitude of the acoustic vibration, to advancing/receding metastable state and to the equilibrium state. The common idea is that in the metastable advancing or receding states, the energy barriers toward equilibrium are

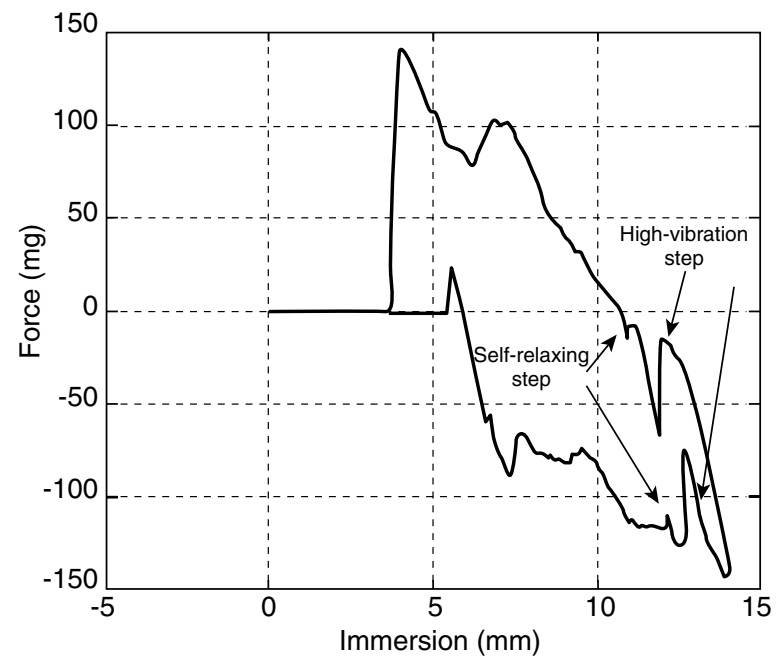

Figure 4

The comparison of the effects of spontaneous relaxation and vibration-induced relaxation in the case of PP samples in water.

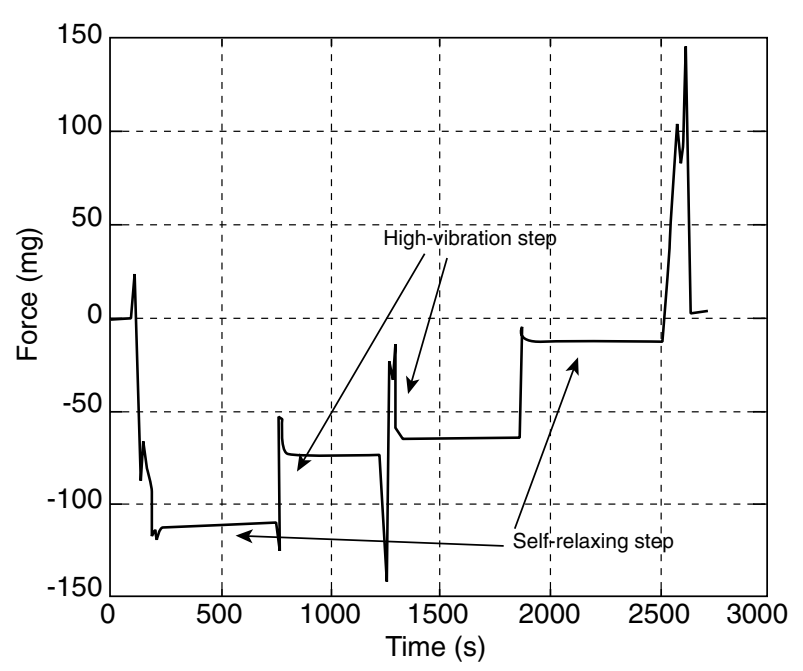

Figure 5

The experiment of Figure 4 in the force $v s$. time representation. 
lower than those existing in the lowest equilibrium state; so we can expect that the same input of acoustic energy has greater effects in the metastable states than in the equilibrium one. This has been fully confirmed by our experiments.

Six increasing amplitudes in advancing and six decreasing in receding at the same resonant frequency have been supplied to the system; the vibration amplitude ranged from 0.2 to $1.2 \mathrm{~V}$. In all cases, the system moved to a more stable state; only the highest amplitude, however, was able to move it in the lowest one, corresponding to the equilibrium state. In all cases, the system moved of a quantity corresponding to a force variation greater than 20 dyn and until 60 dyn (Fig. 6).

In a second experiment, the same amplitude was supplied to a system which was previously moved to the equilibrium state; the vibration cycle was similar to the one used in the first experiment (15" self-relaxing, 15" high vibration, 30" freezing, 30" self-relaxing). At the equilibrium, the system was stressed with three consecutive impulses composed of a 15 ", $0.2 \mathrm{~V}$ amplitude, followed by a 15 " self-relaxing time. Then, a new equilibrium was reached and the system was stressed with three impulses of $0.4 \mathrm{~V}$ amplitude and so on up to $1.2 \mathrm{~V}$, for both the advancing and receding phases. The system was not able to move away from the equilibrium state for a distance corresponding to a force variation greater than 10 dyn (Fig. 7).

Near the extreme value of zero degree contact angle, corresponding to the complete wetting of the surface by the liquid, some peculiar behaviors can be obtained. An RFplasma-cleaned glass plate can be a sample able to show these situations. One can see (Fig. 8a) that during the vibration, the force level attains levels "higher" than those corresponding to a zero contact angle; these states are obtained only as "instantaneous" states, which in this situation correspond only to the three indicated points.

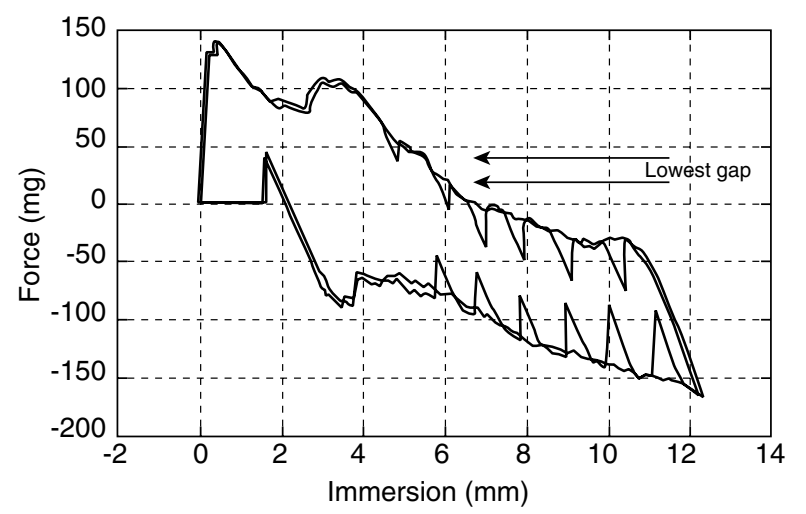

Figure 6

The effects of different amplitudes of acoustic vibrations along the advancing and receding steps. The lowest gap obtained is greater than each gap obtained in the case of equilibrium stage.

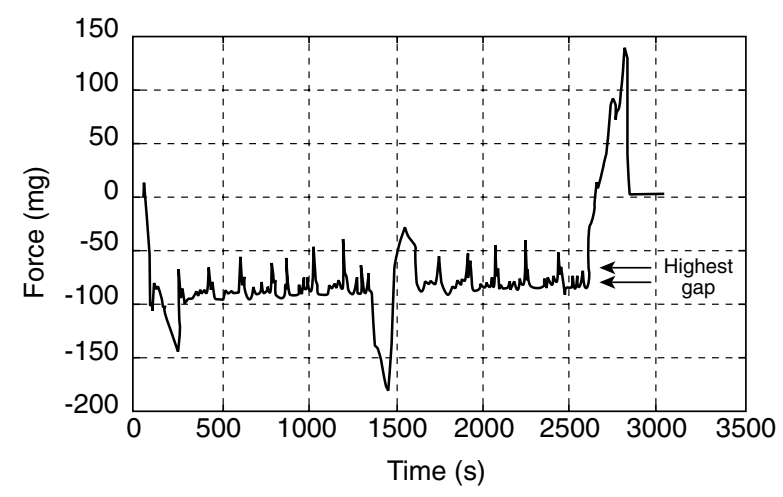

Figure 7

The effects of different amplitudes of acoustic vibrations in the equilibrium phase. The representation is force $v s$. time. The highest gap is lower than each gap of the previous image.
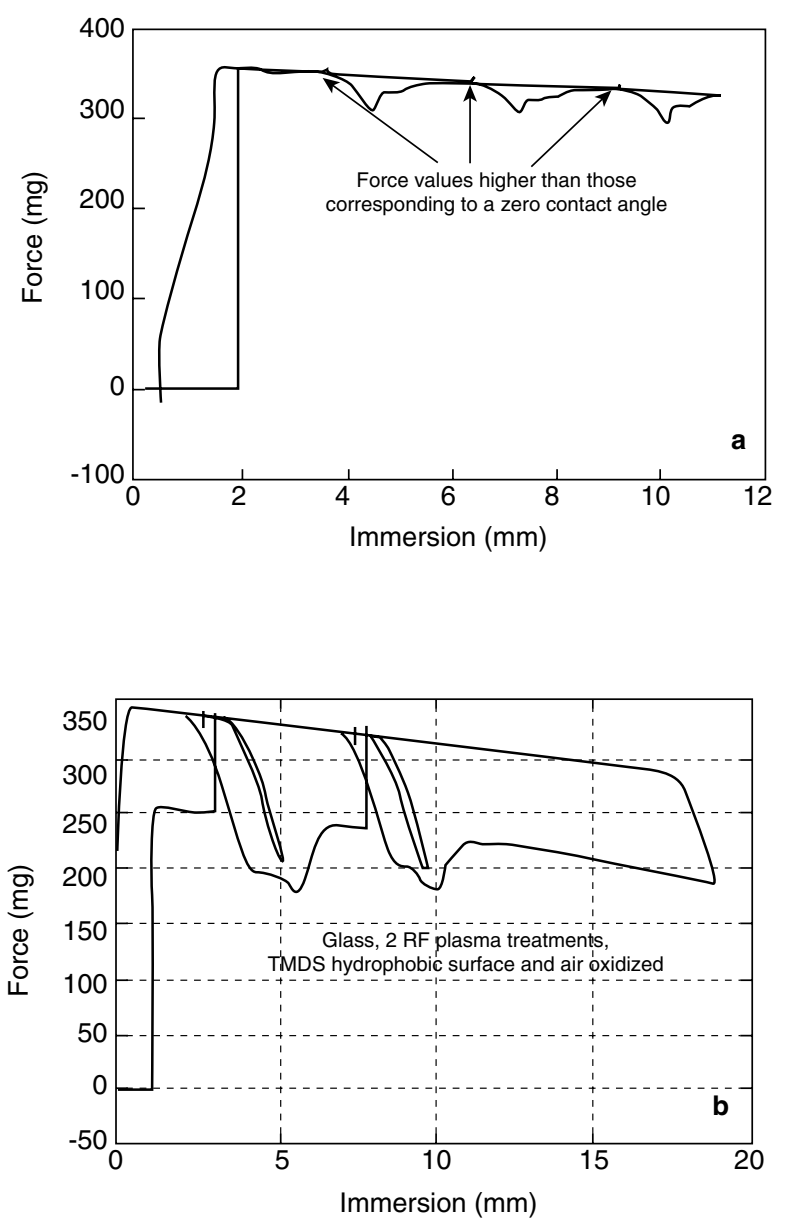

Figure 8

(a) The effect of vibrations in the case of a perfectly wetted sample. The portions of force higher than the receding run are strongly unstable.

(b) RF-plasma-treated samples whose receding portion corresponds to the equilibrium meniscus, due to the formation of an adsorbed liquid film. 
In this experiment, it is also interesting to note how the force, immediately after the vibration phase, during the following new advancing, firstly reduces and then increases again until it acquires the value corresponding to a "zero" contact angle, immediately before the new vibration phase.

This reduction of the force, corresponding to an increase of the contact angle, can be due to the local decrease of the surface free energy of glass due to some wetting effect (RFplasma-induced degradation?). However, further experiments are necessary to clarify this point.

A second case is shown in Figure 8b. A glass surface RF plasma treated by TMDS and then reoxidized by a second RF air plasma treatment is immersed in water. As one can see, the equilibrium values correspond in this case to the receding values, $\theta_{\text {equ }}=0$. This is probably due to the ability of polymer surface to definitely adsorb a water film, changing its structure and/or orientation. Once again, a new immersion in the same zone corresponds to a higher angle, due to the spontaneous elimination of strongly oxidized portion of the surface.

A set of measurement has been performed on the PP samples at different speeds in liquid water; the results are reported in Table 1 and Figure 9. As one can see, the advancing and receding angles do not vary significantly until the speed of $250 \mu \mathrm{m} / \mathrm{s}$ (which is the maximum one for the Cahn 322 microbalance) is attained. The hysteresis increases significantly, but the equilibrium value appears substantially constant in the limits of the standard deviation. In this case, it is worth of note that the number of points used for the determination of the equilibrium value is an important element; its increase to 16 points makes the value of the equilibrium angle be nearer to the mean value (Table 1 ).

TABLE 1

PP samples, data obtained by using vibration technique, $R_{a}=48 \mathrm{~nm}$ : values of the advancing, receding and equilibrium contact angles and their cosines obtained in Milli-Q water for PP samples at different speeds

(the arithmetic mean of cosines and the corresponding angles are also reported)

\begin{tabular}{l|c|c|c|c|c|c}
\hline Liquid & $\begin{array}{c}\text { Frequency } \\
(\mathbf{H z})\end{array}$ & $\begin{array}{c}\text { Speed } \\
(\boldsymbol{\mu m} / \mathbf{s})\end{array}$ & $\begin{array}{c}\theta_{\text {adv }} \\
\left(^{\circ}\right)\end{array}$ & $\begin{array}{c}\theta_{\text {rec }} \\
\left({ }^{\circ}\right)\end{array}$ & $\begin{array}{c}\theta_{\text {equ }} \\
\left(^{\circ}\right)\end{array}$ & $\begin{array}{c}\theta_{1 / 2} \mathbf{c o s}(a+r) \\
\left({ }^{\circ}\right)\end{array}$ \\
\hline Water & & 20 & 108.7 & 80.2 & 95.4 & 94.3 \\
6 points & 54 & 40 & 109.9 & 81.0 & 98.9 & 95.3 \\
6 points & 54 & 80 & 108.8 & 80.4 & 97.7 & 94.5 \\
6 points & 54 & 165 & 108.5 & 80.2 & 98.1 & 94.2 \\
6 points & 54 & 250 & 112.7 & 71.4 & 93.2 & 91.9 \\
6 points & 54 & & & & $96.7 \pm 2.3$ & $94.0 \pm 1.3$ \\
16 points & 54 & 20 & 109.1 & 81.2 & 98.1 & 95.0 \\
Diiodo- & 54 & 20 & 69.2 & 21.4 & 42.8 & 38.6 \\
methane & & & & & & \\
\hline
\end{tabular}

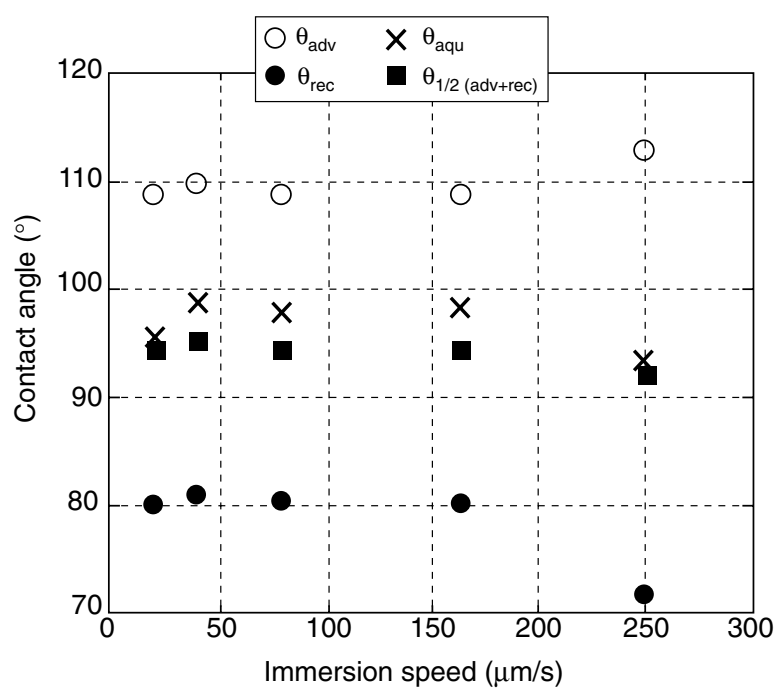

Figure 9

The advancing, receding and equilibrium contact angles of water on PP at different speeds. Also, the values of the angles obtained from the mean of advancing and receding cosines are shown.

We have also analyzed samples whose surface was artificially modified, introducing higher energy portions or lower energy portions in surfaces previously homogeneous and of lower or higher energy, respectively. Two kinds of samples have been analyzed:

- cold plasma-cleaned glass sprayed with different quantities of painting drops;

- RF-plasma-hydrophobized glasses increasingly reoxidized by air RF plasma.

The results obtained with these samples are shown in Tables 2 and 3. Plotting all the results on a graph of $\cos \theta_{\text {equ }}$ vs. $0.5\left(\cos \theta_{\text {adv }}+\cos \theta_{\text {rec }}\right)$, it appears that the $y=x$ curve is a good fitting one. Only three data, which pertain to the only macroscopically inhomogeneous samples, made by the first type of drop coating, deviate from the straight line (Fig. 10). Moreover, the evaluation of the percentage of the different portions of the surfaces, made by Equation (4), is in good agreement with direct "optical" evaluation of the same parameter (Table 2).

These results suggested us an interpretation of these data and of those of Andrieu et al. [23]. In the case of Andrieu et al. data [23], the values obtained using methylene iodide, and in our case, the data obtained on macroscopically inhomogeneous surfaces using water, deviate from the straight line.

Methylene iodide has a surface tension lower than water and closer to that of the PET support but significantly different from that of the sprayed material; water has a surface tension significantly higher than both the PET support and the added material. So, water was less able to "read" the difference between the two components of the inhomogeneous surface. 
TABLE 2

Sprayed glass samples: values of the advancing, receding and equilibrium contact angles and their cosines obtained in Milli-Q water for RF-plasma-sprayed samples at different speeds (the arithmetic mean of cosines and the corresponding angles are also reported)

\begin{tabular}{l|c|c|c|c|c}
\hline & $\theta_{\text {adv }}$ & $\theta_{\text {rec }}$ & $\theta_{\text {equ }}$ & $\theta_{1 / 2 \cos (a+r)}\left({ }^{\circ}\right)$ & \% of coating (optical) \\
\hline Clean glass & 0 & 0 & $0(0 \%)$ & 0 & 0 \\
Sample 1 & 39 & 10 & $18(7 \%)$ & 28 & 2 \\
Sample 2 & 64 & 14 & $25(14 \%)$ & 45 & 14 \\
Sample 3 & 82 & 41 & $53(61 \%)$ & 64 & 54 \\
Fully coated & 91 & 55 & $70(100 \%)$ & 74 & 100 \\
\hline
\end{tabular}

TABLE 3

Cold plasma reoxidized samples: values of the advancing, receding and equilibrium contact angles and their cosines obtained in Milli-Q water for RF-plasma-treated samples at different reoxidation degrees (the arithmetic mean of cosines and the corresponding angles are also reported)

\begin{tabular}{|c|c|c|c|c|c|c|c|c|}
\hline Sample & $\theta_{\text {adv }}$ & $\cos \theta_{\mathrm{adv}}$ & $\theta_{\text {rec }}$ & $\cos \theta_{\text {rec }}$ & $\theta_{\text {equ }}$ & $\cos \theta_{\text {equ }}$ & $0.5 \cos (\mathrm{adv}+\mathrm{rec})$ & $\theta_{1 / 2 \cos (a+r)}\left({ }^{\circ}\right)$ \\
\hline $0 / 1^{(1)}$ & 106.1 & -0.2773 & 90.6 & -0.0105 & 99.2 & -0.1599 & -0.1439 & 98.3 \\
\hline $0 / 2$ & 108.1 & -0.3107 & 94.1 & -0.0715 & 102.0 & -0.2079 & -0.1911 & 101.0 \\
\hline $0 / 3$ & 108.1 & -0.3107 & 90.0 & 0.0000 & 100.6 & -0.1840 & -0.1553 & 98.9 \\
\hline $1 / 2^{(2)}$ & 80.2 & 0.17021 & 63.9 & 0.43994 & 70.6 & 0.33216 & 0.30507 & 72.2 \\
\hline $1 / 3$ & 83.0 & 0.12187 & 63.5 & 0.44620 & 70.1 & 0.34038 & 0.28403 & 73.5 \\
\hline $2 / 1^{(3)}$ & 57.6 & 0.53583 & 37.1 & 0.79758 & 46.7 & 0.68582 & 0.66671 & 48.2 \\
\hline $2 / 2$ & 61.3 & 0.48022 & 43.8 & 0.72176 & 51.1 & 0.62796 & 0.60099 & 53.1 \\
\hline $2 / 3$ & 62.7 & 0.45865 & 46.8 & 0.68455 & 54.4 & 0.58212 & 0.57160 & 55.1 \\
\hline $3 / 1^{(4)}$ & 45.4 & 0.70215 & 25.1 & 0.90557 & 34.9 & 0.82015 & 0.80386 & 36.5 \\
\hline $3 / 2$ & 43.5 & 0.72537 & 30.1 & 0.86515 & 37.6 & 0.79229 & 0.79526 & 37.3 \\
\hline $3 / 3$ & 45.8 & 0.69717 & 30.2 & 0.86427 & 37.9 & 0.78908 & 0.78072 & 38.7 \\
\hline
\end{tabular}

In our case, the use of a clean glass support coated by macroscopically inhomogeneous drops causes that even water is able to "read" the difference between the two components of the surface. On the other hand, RF-plasmamodified surfaces are not macroscopically inhomogeneous.

It is worth of note that in both cases roughness does not appear to play a significant role; in fact, samples with the same roughness can be or not on the straight line $y=x$.

As a conclusion, Equation (2) is valid for homogeneous surfaces or in the case when the used liquid is not able to detect differences among the different surface portions. This corresponds to a single symmetrical $\Delta F(\cos \theta)$ curve.

Suppose now to have two macroscopically distinct portions on the surfaces and to use a liquid able to read this difference. We will have two distinct $\Delta F(\cos \theta)$ functions. The advancing portion of the right curve and the receding portion of the left one, which represent the lowest states in advancing and receding respectively, will give a global asymmetric function, made of the combination of the two starting curves. In this case, the equilibrium value of cosine will not be equivalent to the mean of the advancing and receding cosines. However, in general, it is possible to prove that even a single curve can be unsymmetrical, a case considered in the mathematical appendix.

What is the size limit to consider a certain surface macroscopically heterogeneous? This question should be studied in future. However, from existing data, it appears that while hysteresis can be produced by a heterogeneous structure made of micro- or even nano-patches, the existence of patches at least greater than few microns is necessary to get an unsymmetrical functional of energy for an interfacial system. 


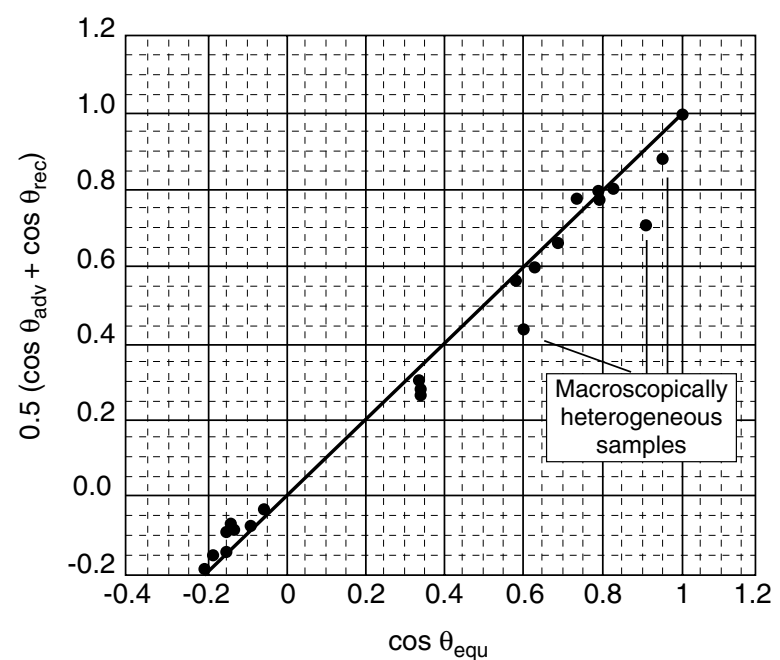

Figure 10

The experimental data of Tables 1,2 and 3 in the same representation used for Andrieu et al. data [23]. Only the results obtained for macroscopically heterogeneous samples deviate sensibly from the fitting $y=x$ line.

A final consideration is about the role of the equilibrium contact angle in the field of surface energetics; the use of the advancing contact angle to evaluate the surface free energy and its components is a common practice. This fact is obviously a source of errors and approximations. As a general consequence, the values of the surface free energy of all the polymer solids can be significantly "underestimated". Due to the ill-conditioning and to the mathematical problems of the acid-base theory $[6,30]$ it has a poor meaning to evaluate the surface free energy of the PP from the very few data we have measured. However, only as an example of the possible effects of the previous considerations, we have recalculated the surface free energy of PP from the data of Table 2; from the advancing, the result is $23 \mathrm{mN} / \mathrm{m}$, while from the equilibrium data, the result is $37 \mathrm{mN} / \mathrm{m}$. This should induce us to reconsider the common evaluation of these quantities, with the warning due to the low precision until now obtained with the present method.

\section{CONCLUSIONS}

In the present paper, a new method has been presented to obtain an equilibrium contact angle with a simple modification of the Wilhelmy microbalance device. The supply of acoustic energy to the liquid at a frequency which induces resonant capillary waves at the liquid surface and the following reduction of the amplitude of the vibration allow the meniscus to move from the metastable advancing or receding shape to the stable equilibrium one.

This result has been shown as significantly independent of the initial conditions; it has been possible to confirm the theoretical provision that the energy barriers between metastable states, moving to equilibrium, are lower than the barriers at equilibrium.

The "mean cosine" equation $[23,28]$ has been confirmed on homogeneous surfaces, but has been disproved on macroscopically heterogeneous surfaces. It depends on the symmetry of the functional of energy at the triple phase line. However, it is possible to mathematically prove that this symmetry is not strictly valid, even in simple cases.

As a consequence, the use of this or similar experimental methods is strongly recommended to obtain the true equilibrium contact angles on macroscopically heterogeneous surfaces. The "critical" size level to define the "macroscopic" character of heterogeneity should be obtained from a wider and opportune set of experimental data.

The method appears promising also for its simplicity and low cost; future improvements will investigate the development of programs which completely automatize the experiment, increasing the number of points collected at the equilibrium condition.

\section{REFERENCES}

1 Young, T. (1805) An Essay on the Cohesion of Fluids. Phil. Trans. Roy. Soc. London, 95, 65-87.

2 Johnson, Jr., R.E. and Dettre, R. (1969) Wettability and Contact Angles, in Surface and Colloid Science, Matijevic, E. (ed.), Vol. 2, Wiley.

3 Good, R.J. (1993) Contact Angle, Wettability and Adhesion: A Critical Review, in Contact Angle Wettability and Adhesion, Mittal, K.L. (ed.), VSP BV, The Netherlands, 336.

4 Saiz, E., Tomsia, A.P. and Cannon, R.M. (1998) Ridging Effects on Wetting and Spreading of Liquids and Solids. Acta Mater., 46, 2349-2361.

5 Andrade, J.D., Lee, M.S. and Gregonis, D.E. (1985) The Contact Angle and Interface Energetics, in Surface and Interfacial Aspects of Biomedical Polymers, Andrade, J.D. (ed.), Vol. 1, Plenum Press, New York.

6 Della Volpe, C. and Siboni, S. (1997) Some Reflections on Acid-Base Solid Surface Free Energy Theories. J. Coll. Interf. Sci., 195, 121-136.

7 Cassie, A.B.D. and Baxter, S. (1944) Wettability on Porous Surfaces. Trans. Faraday Soc., 40, 546-551.

8 Cassie, A.B.D. (1948) Contact Angles. Discussion Faraday Soc., 3, 11-16.

9 Shuttleworth, R. and Bailey, C.L.J. (1948) The Spreading of a Liquid over a Rough Solid. Discussion Faraday Soc., 3, 1622.

10 Johnson, Jr., R.E. and Dettre, R. (1964) Contact Angle Hysteresis. III. Study of an Idealized Heterogeneous Surface. J. Phys. Chem., 68, 1744-1750.

11 Jordan, D.O. and Lane, J.E. (1964) A Thermodynamic Discussion of the Use of a Vertical Plate Balance for the Measurement of Surface Tension. Aust. J. Chem., 17, 7-15.

12 Neumann, A.W. and Good, R.J. (1972) Thermodynamics of Contact Angles. I. Heterogeneous Solid Surfaces. J. Coll. Interf. Sci., 28, 341-358. 
13 Li, D. and Neumann, A.W. (1996) Thermodynamic Status of Contact Angles, in Applied Surface Thermodynamics, Neumann, A.W. and Spelt, J.K. (eds.), Marcel Dekker, New York.

14 Huh, C. and Mason, S.G. (1977) Effects of Surface Roughness on Wetting. J. Coll. Interf. Sci., 60, 11-38.

15 Joanny, J.F. and de Gennes, P.G. (1984) A Model for Contact Angle Hysteresis. J. Chem. Phys., 81, 552-562.

16 Starov, V.M. (1992) Equilibrium and Hysteresis Contact Angles. Adv. Coll. Interf. Sci., 39, 147-173.

17 Decker, E.L., Frank, B., Suo, Y. and Garoff, S. (1999) Physics of Contact Angle Measurements. Colloids and Surf. $A, \mathbf{8 6}, 177-188$.

18 Öpick, U. (2000) Contact Angle Hysteresis Caused by a Random Distribution of Weak Heterogeneities on a Solid Surface. J. Coll. Interf. Sci., 223, 143-166.

19 Del Giudice, G.R.M. (1936) The Bubble Machine for Flotation Testing. Eng. Mining J., 137, 291-294.

20 Fowkes, F.D. and Harkins, W.D. (1940) The State of Monolayers Adsorbed at the Interface Solid-Aqueous Solutions. J. Am. Chem. Soc., 62, 3377-3386.

21 Phillipoff, W., Cooke, S.R.B. and Caldwell, D.E. (1952) Contact Angles and Surface Coverage. Mining Eng., 4, 283-286.

22 Smith, T. and Lindberg, G. (1978) Effect of Acoustic Energy on Contact Angle Measurements. J. Coll. Interf. Sci., 66, 363-366.
23 Andrieu, C., Sykes, C. and Brochard, F. (1994) Average Spreading Parameter on Heterogeneous Surfaces. Langmuir, 10, 2077-2080.

24 Decker, E.L. and Garoff, S. (1996) Using Vibrational Noise to Probe Energy Barriers Producing Contact Angle Hysteresis. Langmuir, 12, 2100-2110.

25 Johnson, Jr., R.E. and Dettre, R. (1965) Contact Angle Hysteresis. IV. Contact Angle Measurement on Heterogeneous Surface. J. Phys. Chem., 69, 1507-1514.

26 Schultze, R.D., Possart, W., Kamusewitz, H. and Bischof, C. (1989) Young's Equilibrium Contact Angle on Rough Solid Surfaces. Part I. An Empirical Determination. J. Adhesion Sci. Technol., 3, 39-48.

27 Di Meglio, J.M. (1992) Contact Angle Hysteresis and Interacting Surface Defects. Europhys. Lett., 17, 607-612.

28 Johnson, Jr., R.E. and Dettre, R. (1993) Wetting of Low Energy Surfaces, in Wettability, Berg, J.C. (ed.), Marcel Dekker, New York, 2-71.

29 Wenzel, R.N. (1936) Resistance of Solid Surfaces to Wetting by Water. Ind. Eng. Chem., 28, 988-994.

30 Della Volpe, C. and Siboni, S. (2000) Acid-Base Solid Surface Free Energies and the Definition of Scales in Goodvan Oss-Chaudury Theory. J. Adhesion Sci. Technol., 14, 239-276.

Final manuscript received in November 2000 


\section{APPENDIX}

\section{A Model of Heterogeneous Planar Solid Surface}

We consider a three-phase system consisting of a heterogeneous solid, a liquid and vapor affected by gravity (with acceleration g). All the surface heterogeneities are assumed not to vary in the horizontal direction along the solid, as in the Neumann-Good model $[12,13]$, but apart from this they are not subjected to further constraints, nor they must take a particular form (like the two-step piecewise constant and periodic behavior of the Neumann-Good model).

Denoted with $y$ and $z$ the horizontal and vertical coordinates along the solid, and with $x$ the horizontal coordinate perpendicular to the same surface, the shape of the equilibrium surface of a liquid with density $\rho$, liquid-vapor surface tension $\gamma$ and equilibrium contact angle $\theta$ on the solid at the three-phase line can be parameterized as follows:

$$
\left\{\begin{array}{l}
x=\frac{1}{\sqrt{c}}\{\alpha-2 \cos (\phi / 2)-\ln [\tan (\phi / 4)]\} \\
z=\frac{1}{\sqrt{c}} 2 \sin (\phi / 2)
\end{array}\right.
$$

where $c=\rho g / \gamma$, the constant $\alpha$ takes the form:

$$
\alpha=2 \cos \left(\frac{\pi}{4}-\frac{\theta}{2}\right)+\ln \left[\tan \left(\frac{\pi}{8}-\frac{\theta}{4}\right)\right]
$$

and $\phi$ is the angle that the line perpendicular to the equilibrium liquid-vapor interface at the parameterized point forms with the upwards-oriented vertical axis. The $x y$-plane and the liquid-vapor interface overlap at a suitable distance from the solid specimen.

If we confine ourselves to the only states where the liquidvapor interface satisfies Laplace equation with an arbitrary "formal" contact angle $\theta$ and if we assume as zero free energy reference state the one where the liquid-vapor interface is totally aligned with the $x y$-plane, the free energy of the system per unit length in the $y$-direction is a sum of three contributions:

- a gravitational contribution:

$$
\begin{aligned}
\Delta F_{\mathrm{g}} & =\frac{1}{2} \rho \mathrm{g} \int_{x=0}^{x=+\infty} z^{2} \mathrm{~d} x \\
& =\frac{\gamma}{3} \sqrt{\frac{2}{c}}[(2-\sin \theta) \sqrt{1+\sin \theta}-\sqrt{2}]
\end{aligned}
$$

- a liquid-vapor surface tension contribution:

$$
\Delta F_{l v}=\gamma \int_{x=0}^{x=+\infty}(\mathrm{d} s-\mathrm{d} x)=\gamma \sqrt{\frac{2}{c}}[\sqrt{2}-\sqrt{1+\sin \theta}]
$$

on having denoted with $\mathrm{d} s$ the infinitesimal length at a point of the intersection of the $x z$-plane with the liquidvapor interface;

- a solid-liquid surface free energy contribution. Relative to the reference state, if $\gamma_{s l}$ and $\gamma_{s v}$ are the surface solid-liquid and solid-vapor free energies respectively, the variation of free energy is obtained by integrating over the meniscus surface in contact with the solid:

$$
\Delta F_{s l}=-\int_{z=0}^{z=h}\left(\gamma_{s v}-\gamma_{s l}\right) \mathrm{d} z=-\gamma \int_{z=0}^{z=h} \frac{\gamma_{s v}-\gamma_{s l}}{\gamma} \mathrm{d} z
$$

where for any $\theta \in[0, \pi]$ the height $h$ of the meniscus is:

$$
h=\sqrt{\frac{2}{c}} \frac{\cos \theta}{\sqrt{1+\sin \theta}}
$$

The equilibrium contact angle $\theta_{e}$ is defined according to Young Equation (1) provided that $-1 \leq\left(\gamma_{s v}-\gamma_{s l}\right) / \gamma \leq 1$. Heterogeneity along the vertical direction means of course that $\gamma_{s v}-\gamma_{s l}$ can be any integrable function of the variable $z$, so that Equation (A.5) becomes:

$$
\begin{aligned}
\Delta F_{s l} & =-\left.\gamma \int_{z=0}^{z=h} \frac{\gamma_{s v}(z)-\gamma_{s l}(z)}{\gamma} \mathrm{d} z\right|_{h=\sqrt{2 / c} \cos \theta / \sqrt{1+\sin \theta}} \\
& =-\gamma \sqrt{\frac{2}{c}} \int_{0}^{\cos \theta / \sqrt{1+\sin \theta}} \Gamma(\xi) \mathrm{d} \xi
\end{aligned}
$$

where:

$$
\Gamma(\xi)=\frac{1}{\gamma}\left[\gamma_{s v}\left(\sqrt{\frac{2}{c}} \xi\right)-\gamma_{s l}\left(\sqrt{\frac{2}{c}} \xi\right)\right]
$$

may be, for instance, any piecewise continuous function of the real variable $\xi \in[-1,+1]$.

The sum of Equations (A.3), (A.4), (A.6) finally provides the total variation of the free energy relative to the reference state:

$$
\begin{aligned}
\Delta F(\theta)= & \gamma \sqrt{\frac{2}{c}} \\
& \left\{\frac{1}{3}\left[2 \sqrt{2}-(1+\sin \theta)^{3 / 2}\right]-\int_{0}^{\cos \theta / \sqrt{1+\sin \theta}} \Gamma(\xi) \mathrm{d} \xi\right\}
\end{aligned}
$$

The metastable states of the system, for any heterogeneity distribution modelled by $\Gamma(\xi)$, are the local minima of $\Delta F(\theta)$ and if $\Gamma(\xi)$ is continuous they may be searched among the solutions of the equation $\Gamma[\cos \theta \sqrt{1+\sin \theta}]=\cos \theta$, which individuates all the critical points of $\Delta F(\theta)$. The mean 
value $\mu$ of $\Gamma(\xi)$ along the sample surface accessible to the meniscus is:

$$
\mu=\frac{1}{2} \int_{-1}^{+1} \Gamma(\xi) \mathrm{d} \xi
$$

and allows us to rewrite Equation (A.8) in the equivalent form:

$$
\Delta F(\theta)=\gamma \sqrt{\frac{2}{c}}\left[C_{\mu}(\theta)+R(\theta)\right]
$$

on having defined in $\theta \in[0, \pi]$ the functions below:

$$
\begin{aligned}
& C_{\mu}(\theta)=-\mu \frac{\cos \theta}{\sqrt{1+\sin \theta}}+\frac{1}{3}\left[2 \sqrt{2}-(1+\sin \theta)^{3 / 2}\right] \\
& R(\theta)=-\int_{0}^{\cos \theta / \sqrt{1+\sin \theta}}[\Gamma(\xi)-\mu] \mathrm{d} \xi
\end{aligned}
$$

If $-1 \leq \mu \leq 1, C_{\mu}(\theta)$ reaches its absolute minimum at $\theta=\theta_{\mu}=\arccos \mu$, as shown in Figure $11 ; \theta_{\mu}$ is the analogous of Cassie-Baxter angle, because:

$$
\cos \theta_{\mu}=\frac{1}{2} \int_{-1}^{+1} \Gamma(\xi) \mathrm{d} \xi \in[-1,+1]
$$

provides the natural generalization of it to a surface where $\Gamma(\xi)$ is not two-step piecewise constant and periodic $(\Gamma(\xi)$ $\mathrm{d} \xi / 2$ represents the contribution to $\cos \theta_{\mu}$ of the solid surface between $\xi$ and $\xi+\mathrm{d} \xi$ ). Whenever $\mu>1$, which may occur if an appreciable portion of the solid surface is characterized by a high surface free energy, the function $C_{\mu}(\theta)$ has no critical point in the interval $0 \leq \theta \leq \pi$ and it takes its absolute minimum at $\theta=0$ (note the correspondence with the case of Figures $8 \mathrm{a}$ and $8 \mathrm{~b}$ ). Similarly, when a significant part of the solid surface has a very low free energy, and thus $\mu<-1$, the absolute minimum of $C_{\mu}(\theta)$ in $0 \leq \theta \leq \pi$ occurs at $\theta=\pi$.

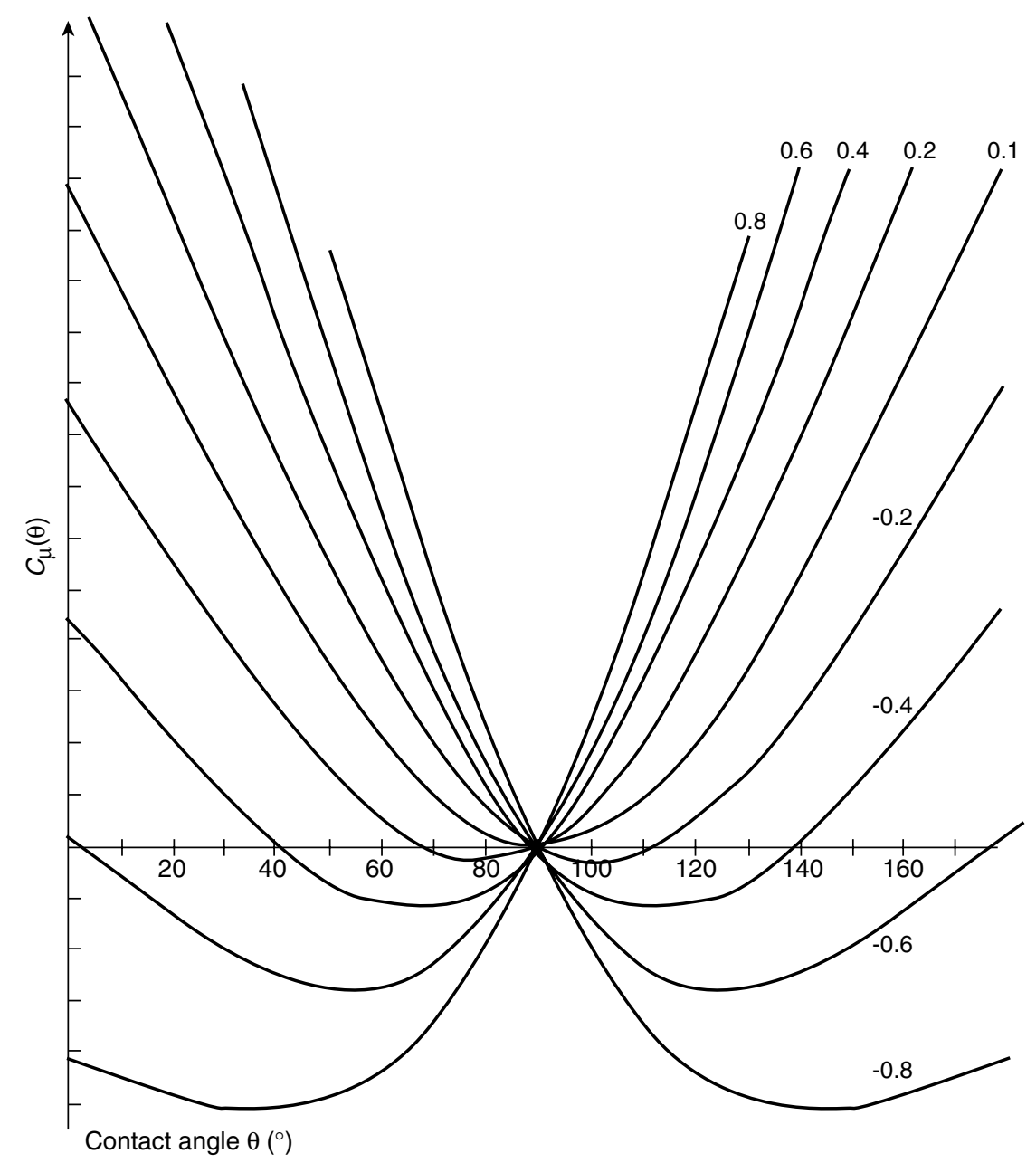

Figure 11

Graph of the function $C_{\mu}(\theta) v s$. the formal contact angle $\theta$ for different values of $\mu$ in the interval $[-1,+1]$. For simplicity's sake, the angle $\theta$ is expressed in degrees. The unique absolute minimum of each curve corresponds to the Cassie-Baxter angle $\theta_{\mu}=\arccos \mu$. 


\section{The Periodic Case}

Suppose that $\Gamma(\xi)$ is a periodic function with period $\Delta \xi<<1$ and mean:

$$
\langle\Gamma\rangle_{\Delta \xi}=\frac{1}{\Delta \xi} \int_{-1}^{+1} \Gamma(\xi) \mathrm{d} \xi
$$

which is easily proved to be almost equal to the value $\mu$ defined by Equation (A.9), so that $\mu \cong\langle\Gamma\rangle_{\Delta \xi}$ for $\Delta \xi<<1$. To a great approximation, we conclude that:

$$
R(\theta)=-\int_{0}^{\cos \theta / \sqrt{1+\sin \theta}}[\Gamma(\xi)-\mu] \mathrm{d} \xi \cong-\int_{0}^{1-2 \theta / \pi}[\Gamma(\xi)-\mu] \mathrm{d} \xi
$$

is in turn almost periodic with approximate period $\pi \Delta \xi / 2$. The graph of the free energy $\Delta F(\theta)$ is then determined by the superposition of the convex graph of $C_{\mu}(\theta)$, with a unique local and absolute minimum at $\theta=\theta_{\mu}$, and of the almost periodic, rapidly varying $R(\theta)$. The typical result is a graph of the form illustrated in Figure 12.

The rapid variation of $R(\theta)$ has some important consequences:

- the absolute minimum of $\Delta F(\theta)$ must always be very close to the absolute minimum $\theta_{\mu}$ of $C_{\mu}(\theta)$, which therefore depends only on $\mu$ or, equivalently, on $\left\langle\Gamma>_{\Delta \xi}\right.$. The thermodynamical equilibrium state of the system is attained approximately at the Cassie-Baxter angle $\theta_{\mu}$, or, when either $\mu>1$ or $\mu<-1$, at $\theta=0$ and $\theta=\pi$ respectively;
- the distribution of local minima is in turn approximately periodic, due to the slow variation of $C_{\mu}(\theta)$ compared with $R(\theta)$. Metastable states are many and almost equidistributed along the $\theta$-axis;

- in the regions of the graph of $\Delta F(\theta)$ where the slowly varying slope of $C_{\mu}(\theta)$ is small, adjacent minima reveal small differences in the free energy. In contrast, portions of the graph with a large slope of $C_{\mu}(\theta)$ are associated with comparatively larger differences in the free energy of close local minima. Where the slope of $C_{\mu}(\theta)$ is more pronounced, random transitions between adjacent metastable states which would result in an increment of the hysteresis angle are strongly hindered. In the opposite case of a small $C_{\mu}(\theta)$ slope, the free energy distribution of metastable states is more favorable to random variations of the hysteresis angle, which may easily increase or decrease.

All of these characteristics are mainly related to $C_{\mu}(\theta)$ and therefore to the Cassie-Baxter angle $\theta_{\mu}$, and do not depend in a significant way on the detailed form of $R(\theta)$.

In the absence of an external source of energy and of external forces, all the local minima of the system free energy can be supposed to behave as local attracting points of the system dynamics, owing to the ubiquitous inner dissipative forces (mainly frictions) acting on it.

According to the simple model discussed here, we can state that the attraction basin of each local minimum simply lies within the interval between the two adjacent local maxima which bracket it. The interval should be possibly

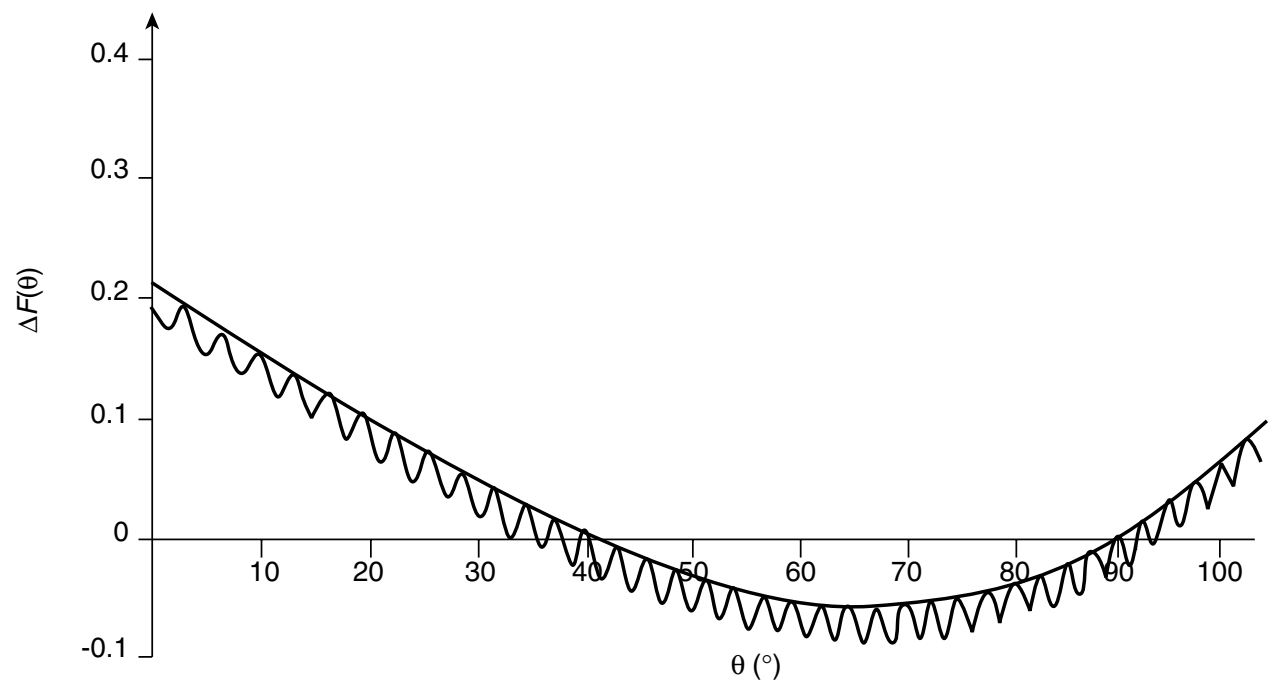

Figure 12

The typical graph of $\Delta F(\theta) v s . \theta$ (degrees) in the periodic case (wavy line), with Cassie-Baxter angle $\theta_{\mu}$. Each local minimum coincides with a metastable state of the system. The graph of $C_{\mu}(\theta)$ is superimposed for comparison. To be noted the proximity of the absolute minimum of $\Delta F(\theta)$ to that of $C_{\mu}(\theta)-\theta_{\mu}$, the almost uniform distribution of local minima and the different free energy spacing of metastable states depending on the slope of $C_{\mu}(\theta)$. 
shrunk so that the total free energy does not exceed the smallest value it takes in the bracketing maxima.

When an external forcing (sound vibration) is turned on, attractivity of local minima is lost owing to the external energy pumping, and the system can more or less freely jump from a minimum to another according to the mean free energy level established by the balance of external forces and inner dissipation.

A sudden turning off of the energy source makes the system spontaneously fall into a local minimum, among the many accessible local equilibria determined by the initial free energy.

Such a transition is obviously due to the only surviving dissipative forces, which restore attractivity of the single local minima. We may expect that the attraction basin of each minimum has a quite complicated and nontrivial structure, so that a prevision of the final metastable equilibrium state attained by the system cannot be made in a reliable way. We can only guess that if the dissipative forces are sufficiently strong, the system will be rapidly attracted by one of the nearest available minima and will stop around it.

The situation changes completely if the abrupt elimination of the external source of energy is replaced by a slow decreasing of vibration amplitude. The energy pumping and depletion due to vibrations and dissipation, respectively, will result in a slowly decreasing mean free energy of the vibrating system and the set of accessible minima will contract more and more, although none of them can be properly considered as an attractor until vibrations persist. In this way the system is guided to move around a smaller and smaller set of local minima, encompassing the absolute free energy minimum. As soon as the forcing amplitude goes to zero and attractivity is then restored, the system will have no other choice than to be attracted by the absolute free energy minimum or by a local minimum very close to it. 